\title{
The frequency of molecular detection of virulence genes encoding cytolysin A, high-pathogenicity island and cytolethal distending toxin of Escherichia coli in cases of sudden infant death syndrome does not differ from that in other infant deaths and healthy infants
}

\author{
Amanda R. Highet, ${ }^{1,2}$ Anne M. Berry, ${ }^{1}$ Karl A. Bettelheimt \\ and Paul N. Goldwater ${ }^{1,2}$ \\ 'Department of Microbiology and Infectious Diseases, SA Pathology, Women's and Children's \\ Hospital, 72 King William Road, North Adelaide, South Australia, Australia \\ ${ }^{2}$ University of Adelaide Discipline of Paediatrics, North Adelaide, South Australia, Australia
}

Correspondence

Amanda R. Highet

amanda.highet@adelaide.edu.au

Received 24 July 2008

Accepted 10 November 2008

\section{INTRODUCTION}

Escherichia coli is of particular interest to sudden infant death syndrome (SIDS) research because early and persistent colonization of the bowel of neonates renders it sufficiently common to fit the age distribution of SIDS. To date, several studies have identified a link between toxin-secreting strains of E. coli and SIDS, notably independent studies by Bettelheim et al. (1990) and Bettiol et al. (1994) who both found toxigenic E. coli in a

tPresent address: Flat 5, Rosedale Lodge, 220 Chase Side, London N14 4PH, UK (Retired).

Abbreviations: APEC, avian pathogenic E. coli; CDT, cytolethal distending toxin; $C N F$, cytotoxic necrotizing factor; ETEC, enterotoxigenic $E$. coli; $\mathrm{HPI}$, high-pathogenicity island; SIDS, sudden infant death syndrome; STEC, Shiga toxin-producing E. coli; UPEC, uropathogenic E. coli; VT+, verotoxigenic.

A supplementary table and two supplementary figures are available with the online version of this paper. high proportion of SIDS infants and less often in healthy comparison babies. The E. coli found in these instances were generally of different serotypes from those expected to be isolated from infants (Bettiol et al., 1994); the isolates from SIDS infants, but not healthy infants, were lethal to mice (Bettelheim et al., 1992a) and frequently induced subtle but distinct changes in vero cell monolayers (Bettelheim et al., 1995). The presence of stx genes in the verotoxigenic $(\mathrm{VT}+)$ isolates could not be confirmed by PCR at the time of Bettelheim's study and it remains unknown whether the VT+ strains described in 1995 were non-stx verotoxigenic. Prolonged storage or repeated subculture of these isolates may have rendered their stx-1 and -2 genes unstable (Karch et al., 1992), as subsequent testing by PCR revealed. Despite these findings, neither Shiga toxin nor heat-labile enterotoxin has been attributed to the causation of SIDS, which is accompanied by a lack of compatible pathological evidence that would reflect the effects of these toxins. 
Human uropathogenic E. coli (UPEC) and avian pathogenic E. coli (APEC) both belong to the extra-intestinal pathogenic E. coli subgroup and share overlapping serotypes and phylogenetic and virulence traits. Human UPEC strains have been shown to share pathogenicityrelated genes with APEC strains, particularly those located on the high-pathogenicity island (HPI), which facilitate the uptake of iron and thus survival of the bacterium in extraintestinal body sites. HPI is found in extra-intestinal E. coli strains associated with urinary tract infection and sepsis (Karch et al., 1999). Interestingly, the predominant E. coli isolates found in the intestinal tract of SIDS infants are typically extra-intestinal serotypes and are more consistent with UPEC (Bettelheim et al., 1995) and thus possibly APEC. APEC produce a high mean lesion score for lethality in 1-day-old chicks, perhaps similar to the high lethality of SIDS E. coli to mice, whereas those isolated from healthy infants were not lethal. Also, the reporting of coliforms in extra-intestinal sites such as the upper respiratory tract (Gilbert et al., 1992) and, more recently, E. coli in normally sterile sites during post-mortem investigation of sudden unexpected death in infancy (Weber et al., 2008; Goldwater, 2008), including SIDS, may be related to the effects of such islands as the HPI.

Cytolethal distending toxin (CDT), encoded by clyA, is produced by a number of pathogens including some of the enteric bacteria such as E. coli, Shigella dysenteriae and Campylobacter spp. and also some APEC. CDTs are characterized by their ability to arrest the target cell cycle irreversibly at the $\mathrm{G}_{2} / \mathrm{M}$ transition (Elwell et al., 2001) where the cells acquire a characteristic distended morphology. Previous studies have found 'functional' clyA (intact without deletions 1 and 2) in E. coli pathogroups causing enteric disease, including Shiga toxin-producing E. coli (STEC), but not in extra-intestinal strains (Ludwig et al., 2004). As diarrhoea is a known risk factor for SIDS, the role of these relatively common toxins warrants investigation.

This study aimed to discern whether the apparent higher frequency of pathogenic E. coli genotypes in SIDS infants holds true for the virulence factors HPI, STEC-type (functional) cytolysin A and CDT.

\section{METHODS}

Strains. Enteric E. coli strains isolated from SIDS and control infants were obtained from the National E. coli Reference Laboratory, Microbiological Diagnostic Unit, Public Health Laboratory, Department of Microbiology and Immunology, University of Melbourne, Melbourne, Australia. Ethical approval had been obtained from the Research Ethics Committee of the Victorian Institute of Forensic Medicine (the institution at which the cases were sampled during autopsy). E. coli isolates from SIDS cases were from samples taken during post-mortem investigation from the intestinal contents of infants who at that time met the 1989 definition of SIDS (Willinger et al., 1991). Control samples were collected during postmortem investigation from the intestinal contents of infants who died from causes other than SIDS (principally traumatic injury, drowning or congenital defects) and from faecal samples from healthy infants attending a day-care centre in Victoria between 1989 and mid 1990 (Bettelheim et al., 1989, 1990, 1995). These strains were involved in previous investigations by Bettelheim et al. (1989, 1990, 1993, 1995) and had been assayed at those times for verocytotoxicity, heat-labile enterotoxin, HeLa cell adherence, haemagglutination, hydrophobicity and cytotoxic necrotizing factor (CNF). Selection of isolates and biochemical identification of these strains have been described previously (Bettelheim et al., 1993). Principally, the single predominant E. coli isolate was taken, and where two isolates of the same serotype were collected, both were tested. On 18 occasions, two isolates of different serotypes were taken. Both isolates were taken into account in the analysis of results, but they were both counted as the result for one infant (i.e. results are in terms of number of infants, not number of isolates). The current investigation was conducted on 181 E. coli strains isolated from 145 SIDS infants and 106 E. coli strains isolated from 28 dead control and 73 healthy infants (henceforth combined as 101 comparison infants). The median age for the SIDS group was 3.5 months with an interquartile range of 3 . The median age for dead control infants was 4 months with an interquartile range of 5.25 . The median age for the healthy infant group was 3 months with an interquartile range of 4 . The percentage of males in the SIDS, dead control and healthy infant groups was 68, 57 and $42 \%$, respectively.

Positive control strains for PCR testing were sourced from stored clinical isolates from cases of haemolytic uraemic syndrome or urinary tract infection in the Department of Microbiology and Infectious Diseases, Women's and Children's Hospital, North Adelaide, Australia. All E. coli strains were grown on blood agar plates for $24 \mathrm{~h}$ at $37^{\circ} \mathrm{C}$.

Strains were tested by PCR for genes coding for intact $c l y A$, without deletion 2, as described by Ludwig et al. (2004), as well as virulenceassociated genes characteristic of APEC or UPEC, namely $c d t B$, a conserved region encoding the B subunit of CDT protein and irp2 which encodes iron-repressible protein 2, an HPI-specific gene.

Oligonucleotide primers. Oligonucleotides synthesized by GeneWorks are given in Supplementary Table S1 (available in JMM Online). ClyA primers amplify clyA when deletion 2 is not present (this was presumed to be functional and was assigned the name clyAf). Forward primer clyA-F matches a region of the $c l y A$ sequence conserved amongst the published sequences for STEC, enterotoxigenic E. coli (ETEC) and UPEC (GenBank accession numbers AY576656, AY576661 and AY576665, respectively). Reverse primer clyA-R was designed with an 8 bp overhang into deletion 2, allowing it to bind to STEC clyAf, but not ETEC or UPEC clyA. Alignment of these primers with three $c l y A$ sequences is shown in Supplementary Fig. S1 (available in JMM Online). The lack of binding to ETEC and UPEC strains was confirmed by sequencing a larger portion of the $c l y A$ gene amplified from strains that amplified accordingly with these primers and confirming the presence of deletion 2. The primer employed for amplification of the larger product was clyAfull-R (Supplementary Table S1). A random selection of E. coli lysates was also screened for $c d t B$, which encodes the B subunit of CDT, as part of a pilot study into the gene's frequency among E. coli from Australian infants. $c d t B$ contains a highly conserved region and so this site was chosen as the target sequence for screening. The primers shown in Supplementary Table S1, published by Toth et al. (2003) allowed multiplex PCR detection of CDT types I, II, III and IV. Analysis of the primers CDTs1 and CDTas2 revealed that $c d t B$ type $\mathrm{V}$ could also be detected (Bielaszewska et al., 2004).

PCR mixes. Each PCR mastermix consisted of $0.1 \mu \mathrm{M} \mathrm{MgCl} 2,1 \times$ Colourless GoTaq Flexi Buffer (Promega), $0.005 \mu \mathrm{M}$ dNTP mix, $0.1 \mu \mathrm{M}$ each primer, 0.5 units GoTaq DNA Polymerase (Promega), $1 \mu \mathrm{l}$ bacterial lysate and water, to a total volume of $12.5 \mu$ l. Primer 
pairs irp2-F, irp2-R and clyA-F, clyA-R could be duplexed in the same reaction to simultaneously detect $\operatorname{irp} 2$ and $c l y A$ (Supplementary Fig. S2, available in JMM Online), in which case the primer concentrations were kept at $0.1 \mu \mathrm{M}$ and the volume of water was adjusted accordingly.

Genes were amplified on a Hybaid OmniGene Thermal Cycler (Hybaid) programmed as follows: initial denaturation $94{ }^{\circ} \mathrm{C} 2 \mathrm{~min}$, 35 cycles of $94{ }^{\circ} \mathrm{C} 30 \mathrm{~s}, 55^{\circ} \mathrm{C} 30 \mathrm{~s}, 72{ }^{\circ} \mathrm{C} 30 \mathrm{~s}$, final extension of $2 \mathrm{~min}$ at $72{ }^{\circ} \mathrm{C}$. Products (280 bp irp2, $204 \mathrm{bp} c l y A f$ and $\left.466 \mathrm{bp} c d t B\right)$ were visualized by ethidium bromide-stained $2 \%$ agarose gel electrophoresis (Supplementary Fig. S2).

\section{RESULTS}

Data from screening of E. coli isolates from SIDS and comparison infants are shown in Table 1. In total, 158 different serotypes were tested. PCR results for a selection of serotypes for which more than one isolate from a SIDS infant was tested are shown in Table 2.

\section{DISCUSSION}

The numbers of $E$. coli isolates containing the genes clyAf, irp2 from the HPI and $c d t B$ from CDT were almost identical between SIDS and comparison infants. This suggests that these genes and their products are not likely to be implicated in the development of SIDS on a purely gene prevalence basis. However, it was interesting to find that of 73 healthy infants, 48 carried irp2-positive (therefore HPI-positive) E. coli and 26 carried clyAfpositive E. coli. Since these infants did not have evidence of E. coli infection at the time of collection, this suggests that carriage of these genes by intestinal $E$. coli is normal and there may be other host or environmental factors that contribute to E. coli disease caused by these agents. These findings were unexpected, yet plausible, considering that Bettelheim et al. (1993) identified a number of $E$. coli strains producing CNF not only in SIDS infants but also in those that died from other causes as well as healthy infants. Nevertheless, given appropriate conditions that could pertain to a SIDS case, any of these toxins could play a role if they acted in combination with the factors that contribute to a SIDS demise, namely exposure to cigarette smoke (Sayers et al., 1995), viral co-infection (Jakeman et al., 1991) and genetic susceptibility (Blackwell et al., 2005; Highet, 2008). Moreover, the proposed toxins could act in concert with bacterial superantigenic products, given that findings indicative of organ shock in SIDS have been observed to be analogous to an E. coli endotoxin-induced mouse model of shock (Blood-Siegfried et al., 2008).

The limitations of this study mainly stem from the infrequency of non-SIDS infant deaths in Australia. Twenty-eight infants that had died of other causes, including drowning or congenital defects, were included in the study as controls to represent post-mortem bacteriology of an infant. The random nature of these deaths meant that they did not have the peak incidence of 2-4 months that is characteristic of SIDS and therefore had a slightly higher median age (4 months) and interquartile range (5.25 months) than SIDS infants (3.5 and 3 months). Healthy infants were more suitably matched for median age (3 months) and interquartile range (4 months); however, any deviations should be considered in the interpretation of these results, as slight differences in gender ratio and age may be masking a subtle increased gene frequency in the SIDS E. coli. Questions can be raised regarding the viability and proportionality of different species between the two sample types. Nevertheless, it has been shown that if $E$. coli is found in the small intestine and/or colon, it is, without fail, also found in the faeces (Bettelheim et al., 1992b). This would therefore support the use of healthy infant faeces as control material in this investigation. Ideally, healthy control infants should be exposed to the same environmental $E$. coli reservoirs as SIDS infants. However, it has been documented that in most cases, the early colonizing E. coli are acquired from an infant's mother (Bettelheim \& Lennox-King, 1976) so perfectly matched case-controls would be particularly difficult in practice, unless of course the SIDS infant had a twin sibling.

According to Schubert et al. (1998), HPI is most prevalent in enteroaggregative E. coli and in strains isolated from blood cultures (93 and $80 \%$, respectively) and rarer in intestinal strains. In a study of 60 E. coli from blood cultures, they found $50(83 \%)$ to be positive for irp2, but only 8 of $25(32 \%)$ E. coli from stool samples from healthy individuals tested positive for irp2 using PCR (Schubert et al., 1998). In this study we have found that the infant population has a high prevalence of carriage of irp2 (64$67 \%)$. These findings may reflect that the infant intestine normally harbours these strains which decrease in preval-

Table 1. Results of PCR screening of $E$. coli isolates for clyAf, irp2 (HPI) and cdtB genes

The table shows the number of infants that tested positive for each gene by PCR (percentage in parentheses). A subset of infants were tested for $c d t B$ in a pilot study of representative serotypes from 84 SIDS and 23 comparison infants.

\begin{tabular}{|llccc|}
\hline & clyAf & irp2 & Both clyAf and irp2 & cdtB \\
\hline SIDS infants $(n=145)$ & $64(44.1)$ & $97(67)$ & $25(17.2)$ & $0 / 84$ \\
Comparison infants $(n=101)$ & $40(39.6)$ & $65(64.4)$ & $20(19.8)$ & $0 / 23$ \\
\hline
\end{tabular}


Table 2. E. coli serotypes commonly isolated from SIDS infants that tested positive for either clyAf or irp2

\begin{tabular}{|c|c|c|c|c|}
\hline Serotype & No. strains tested & No. $\operatorname{irp} 2+$ & No. clyAf+ & Source $^{\star}$ \\
\hline O1.H- & 5 & 5 & 5 & 4 SIDS, $1 \mathrm{HB}$ \\
\hline O1.H7 & 15 & 15 & 2 & $13 \operatorname{SIDS} \dagger, 1 \mathrm{HB}$ \\
\hline O17.H18 & 2 & 0 & 2 & 2 SIDS \\
\hline $\mathrm{O} 2 . \mathrm{H} 1$ & 3 & 2 & 1 & 2 SIDS, $1 \mathrm{NS}$ \\
\hline $\mathrm{O} 2 . \mathrm{H} 15$ & 3 & 3 & 0 & 3 SIDS \\
\hline $\mathrm{O} 2 . \mathrm{H} 18$ & 2 & 2 & 2 & 2 SIDS \\
\hline $\mathrm{O} 2 . \mathrm{H} 6$ & 3 & 3 & 0 & 3 SIDS \\
\hline $\mathrm{O} 2 . \mathrm{H} 7$ & 3 & 3 & 0 & 3 SIDS \\
\hline $\mathrm{O} 25 . \mathrm{H} 1$ & 5 & 5 & 0 & 3 SIDS, $1 \mathrm{NS}, 1 \mathrm{HB}$ \\
\hline $\mathrm{O} 25 . \mathrm{H} 1 / 12$ & 6 & 6 & 0 & 6 SIDS \\
\hline $\mathrm{O} 25 . \mathrm{H} 2$ & 3 & 3 & 3 & $2 \operatorname{SIDS} \dagger$ \\
\hline O4.H5 & 7 & 7 & 1 & 3 SIDS, 1 NS, $3 \mathrm{HB}$ \\
\hline O5.H4/17 & 2 & 0 & 0 & 2 SIDS \\
\hline O6.H- & 10 & 10 & 0 & 5 SIDS, $5 \mathrm{HB}$ \\
\hline O6.H1 & 4 & 4 & 0 & 3 SIDS, $1 \mathrm{HB}$ \\
\hline O6.H31 & 3 & 3 & 1 & $1 \mathrm{SIDS} \dagger, 1 \mathrm{NS}$ \\
\hline O75.H- & 10 & 10 & 1 & 7 SIDS, 2 NS, $1 \mathrm{HB}$ \\
\hline O75.H7 & 3 & 3 & 0 & 2 SIDS, $1 \mathrm{HB}$ \\
\hline O77.H18 & 4 & 1 & 4 & $2 \mathrm{NS}, 2 \mathrm{HB}$ \\
\hline O91.H11/21 & 3 & 0 & 3 & $2 \operatorname{SIDS} \dagger$ \\
\hline Ont.H- & 17 & 10 & 7 & 10 SIDS $\dagger, 3 \mathrm{NS}, 1 \mathrm{HB}$ \\
\hline Ont.H1 & 6 & 6 & 1 & 3 SIDS, $3 \mathrm{HB}$ \\
\hline Ont.H10 & 2 & 0 & 0 & 2 SIDS \\
\hline Ont.H12 & 2 & 1 & 1 & 2 SIDS \\
\hline Ont.H18 & 2 & 2 & 2 & 2 SIDS \\
\hline
\end{tabular}

${ }^{*}$ Source: infant SIDS (SIDS), non-SIDS death (NS) or healthy baby (HB).

$\dagger$ Denotes more than one isolate from a single infant.

ence after infancy. The isolation of a variety of serotypes between individuals in the healthy infant population negates the idea of one strain of irp2-positive E. coli being transmitted among infants from the day-care centre they were attending. Detection of irp2 by PCR appears to be serotype-dependent, as several strains of the same serotype from different infants gave the same result for irp2. On the other hand, clyAf PCR results appeared to be independent of serotype. This trend is evident in the results shown in Table 2.

To summarize, no single E. coli entity can be attributed to the causation of SIDS, because of either a lack of pathological evidence that would reflect the effects of these toxins and/or their equal prevalence in non-SIDS infants. Three new candidate factors, HPI, ClyA and CDT, were tested in this investigation but failed to show a positive correlation with SIDS, instead proving to be common to the infant intestinal flora. Interestingly, detection of HPI was, in most cases, predictable by serotype, where the gene's presence or absence was consistent in many isolates of the same serotype. Despite the lack of associations defined so far, there remains the likelihood that genetic determinants influence the interactions between $E$. coli and the host and so these factors may be part of the multifactorial aspect of SIDS.

\section{ACKNOWLEDGEMENTS}

The authors wish to thank the Foundation for the Study of Infant Deaths (UK) for kindly funding this project.

\section{REFERENCES}

Bettelheim, K. A. \& Lennox-King, S. M. (1976). The acquisition of Escherichia coli by new-born babies. Infection 4, 174-179.

Bettelheim, K. A., Dwyer, B. W., Smith, D. L., Goldwater, P. N. \& Bourne, A. J. (1989). Toxigenic Escherichia coli associated with sudden infant death syndrome. Med J Aust 151, 538.

Bettelheim, K. A., Goldwater, P. N., Dwyer, B. W., Bourne, A. J. \& Smith, D. L. (1990). Toxigenic Escherichia coli associated with sudden infant death syndrome. Scand J Infect Dis 22, 467-476.

Bettelheim, K. A., Pearce, J., Evangelidis, H. \& Goldwater, P. (1992a). A mouse model for sudden infant death syndrome. Paper presented at the 2nd SIDS Family International Conference, Sydney, Australia.

Bettelheim, K. A., Goldwater, P. N., Evangelidis, H., Pearce, J. L. \& Smith, D. L. (1992b). Distribution of toxigenic Escherichia coli serotypes in the intestines of infants. Comp Immunol Microbiol Infect Dis 15, 65-70.

Bettelheim, K. A., Evangelidis, H., Pearce, J. L., Goldwater, P. N. \& Luke, R. K. J. (1993). The isolation of cytotoxic necrotizing factor (CNF)-producing Escherichia coli from the intestinal contents of 
babies who died of sudden infant death syndrome (SIDS) and other causes as well as from the faeces of healthy babies. Comp Immunol Microbiol Infect Dis 16, 87-90.

Bettelheim, K. A., Chang, B. J., Elliott, S. J., Gunzburg, S. T. \& Pearce, J. L. (1995). Virulence factors associated with strains of Escherichia coli from cases of sudden infant death syndrome (SIDS). Comp Immunol Microbiol Infect Dis 18, 179-188.

Bettiol, S. S., Radcliff, F. J., Hunt, A. L. \& Goldsmid, J. M. (1994). Bacterial flora of Tasmanian SIDS infants with special reference to pathogenic strains of Escherichia coli. Epidemiol Infect 112, 275-284.

Bielaszewska, M., Fell, M., Greune, L., Prager, R., Fruth, A., Tschape, H., Schmidt, A. \& Karch, H. (2004). Characterisation of cytolethal distending toxin genes and expression in shiga-toxin producing Escherichia coli strains of non-O157 serogroups. Infect Immun 72, 1812-1816.

Blackwell, C. C., Moscovis, S. M., Gordon, A. E., Al Madani, O. M., Hall, S. T., Gleeson, M., Scott, R. J., Roberts-Thomson, J., Weir, D. M. \& Busuttil, A. (2005). Cytokine responses and sudden infant death syndrome: genetic, developmental, and environmental risk factors. J Leukoc Biol 78, 1242-1254.

Blood-Siegfried, J., Rambaud, C., Nyska, A. \& Germolec, D. R. (2008). Evidence for infection, inflammation and shock in sudden infant death: parallels between a neonatal rat model of sudden death and infants who died of sudden infant death syndrome. Innate Immun 14, 145-152.

Elwell, C., Chao, K., Patel, K. \& Dreyfus, L. (2001). Escherichia coli $\mathrm{CdtB}$ mediates cytolethal distending toxin cell cycle arrest. Infect Immun 69, 3418-3422.

Gilbert, R., Rudd, P., Berry, P., Fleming, P., Hall, E., White, D., Oreffo, V., James, P. \& Evans, J. (1992). Combined effect of infection and heavy wrapping on the risk of sudden unexpected infant death. Arch Dis Child 67, 171-177.

Goldwater, P. N. (2008). Sterile site infection at autopsy in sudden unexpected deaths in infancy. Arch Dis Child Sep 15 [Epub ahead of print].
Highet, A. R. (2008). An infectious aetiology of sudden infant death syndrome. J Appl Microbiol 105, 625-635.

Jakeman, K. J., Rushton, D. I., Smith, H. \& Sweet, C. (1991). Exacerbation of bacterial toxicity to infant ferrets by influenza virus: possible role in sudden infant death syndrome. J Infect Dis 163, 35-40.

Karch, H., Meyer, T., Russmann, H. \& Heesemann, J. (1992). Frequent loss of Shiga-like toxin genes in clinical isolates of Escherichia coli upon subcultivation. Infect Immun 60, 3464-3467.

Karch, H., Schubert, S., Zhang, D., Zhang, W., Schmidt, H., Olschläger, T. \& Hacker, J. (1999). A genomic island, termed highpathogenicity island, is present in certain non-O157 Shiga toxinproducing Escherichia coli clonal lineages. Infect Immun 67, 5994-6001.

Ludwig, A., von Rhein, C., Bauer, S., Hüttinger, C. \& Goebel, W. (2004). Molecular analysis of cytolysin A (ClyA) in pathogenic Escherichia coli strains. J Bacteriol 186, 5311-5320.

Sayers, N. M., Drucker, D. B., Telford, D. R. \& Morris, J. A. (1995). Effects of nicotine on bacterial toxins associated with cot death. Arch Dis Child 73, 549-551.

Schubert, S., Rakin, A., Karch, H., Carniel, E. \& Heesemann, J. (1998). Prevalence of the "high-pathogenicity island" of Yersinia species among Escherichia coli strains that are pathogenic to humans. Infect Immun 66, 480-485.

Toth, I., Herault, F., Beutin, L. \& Oswald, E. (2003). Production of cytolethal distending toxins by pathogenic Escherichia coli strains isolated from human and animal sources: establishment of the existence of a new $c d t$ variant (type IV). J Clin Microbiol 41, 42854291.

Weber, M. A., Klein, N. J., Hartley, J. C., Lock, P. E., Malone, M. \& Sebire, N. J. (2008). Infection and sudden unexpected death in infancy: a systematic retrospective case review. Lancet 371, 18481853.

Willinger, M., James, L. S. \& Catz, C. (1991). Defining the sudden infant death syndrome (SIDS): deliberations of an expert panel convened by the National Institute of Child Health and Human Development. Pediatr Pathol 11, 677-684. 